\title{
Mohoric, Andrea
}

\section{Transfer von Modellversuchsergebnissen - ein Beispiel aus dem Förderschwerpunkt Berufsbildung für nachhaltige Entwicklung}

Haushalt in Bildung \& Forschung 4 (2015) 1, S. 33-39

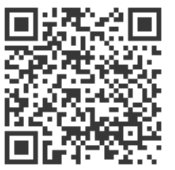

Quellenangabe/ Reference:

Mohoric, Andrea: Transfer von Modellversuchsergebnissen - ein Beispiel aus dem

Förderschwerpunkt Berufsbildung für nachhaltige Entwicklung - In: Haushalt in Bildung \& Forschung 4 (2015) 1, S. 33-39 - URN: urn:nbn:de:0111-pedocs-203612 - DOI: 10.25656/01:20361

https://nbn-resolving.org/urn:nbn:de:0111-pedocs-203612

https://doi.org/10.25656/01:20361

in Kooperation mit / in cooperation with:

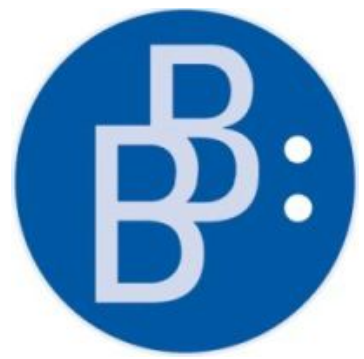

https://www.budrich.de

\section{Nutzungsbedingungen}

Gewährt wird ein nicht exklusives, nicht übertragbares, persönliches und beschränktes Recht auf Nutzung dieses Dokuments. Dieses Dokument ist ausschließlich für den persönlichen, nicht-kommerziellen Gebrauch bestimmt. Die Nutzung stellt keine Übertragung des Eigentumsrechts an diesem Dokument dar und gilt vorbehaltlich der folgenden Einschränkungen: Auf sämtlichen Kopien dieses Dokuments müssen alle Urheberrechtshinweise und sonstigen Hinweise auf gesetzlichen Schutz beibehalten werden. Sie dürfen dieses Dokument nicht in irgendeiner Weise abändern, noch dürfen Sie dieses Dokument für öffentliche oder kommerzielle Zwecke vervielfältigen, öffentlich ausstellen, aufführen, vertreiben oder anderweitig nutzen.

Mit der Verwendung dieses Dokuments erkennen Sie die Nutzungsbedingungen an.

\section{Terms of use}

We grant a non-exclusive, non-transferable, individual and limited right to using this document.

This document is solely intended for your personal, non-commercial use. Use of this document does not include any transfer of property rights and it is conditional to the following limitations: All of the copies of this documents must retain all copyright information and other information regarding legal protection. You are not allowed to alter this document in any way, to copy it for public or commercial purposes, to exhibit the document in public, to perform, distribute or otherwise use the document in public.

By using this particular document, you accept the above-stated conditions of use.

\section{Kontakt / Contact:}

\section{peDOCs}

DIPF | Leibniz-Institut für Bildungsforschung und Bildungsinformation Informationszentrum (IZ) Bildung

E-Mail: pedocs@dipf.de

Internet: www.pedocs.de 


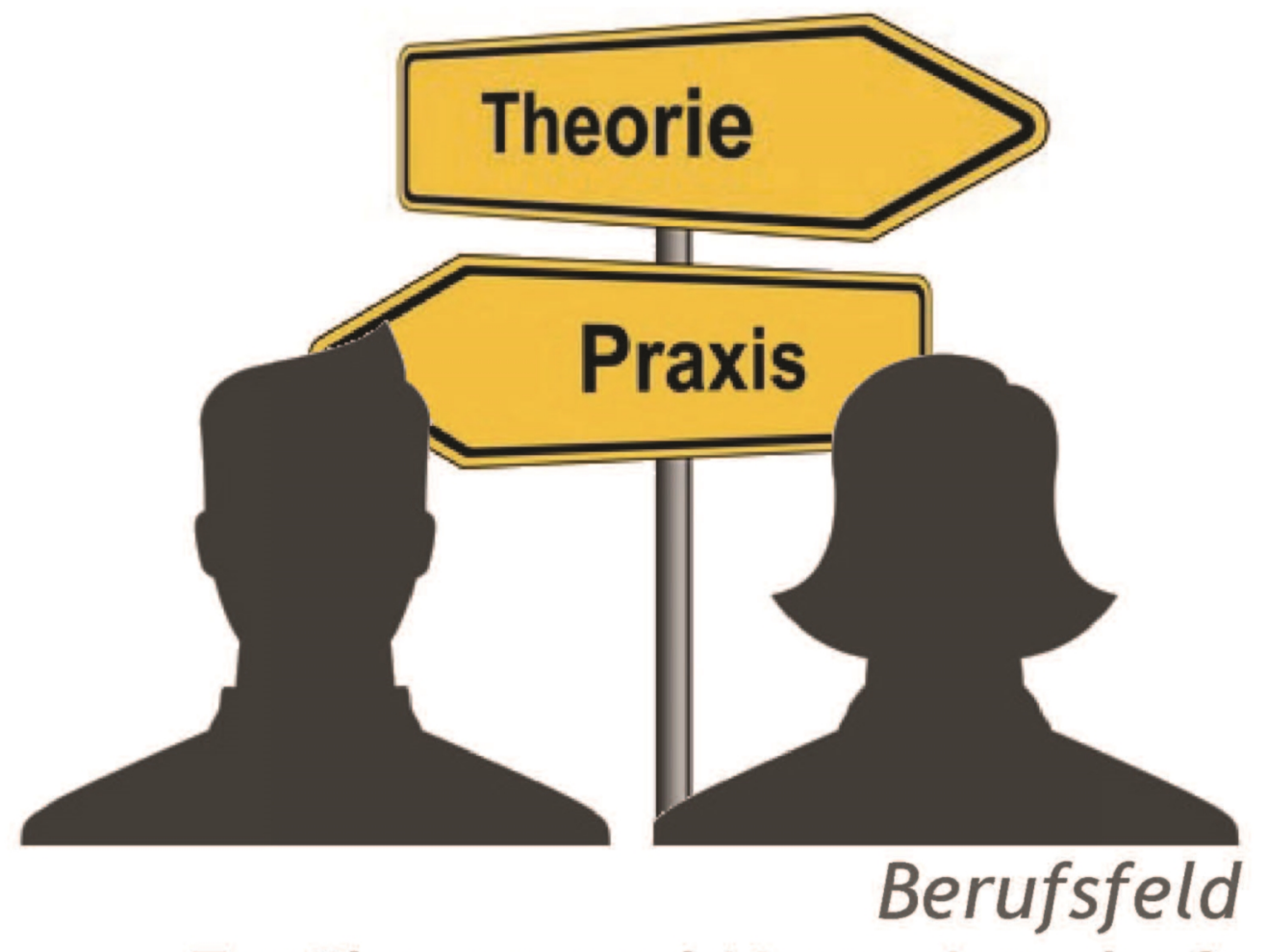

Ernährung und Hauswirtschaft

Transfer zwischen Theorie und Praxis 
Irmhild Kettschau

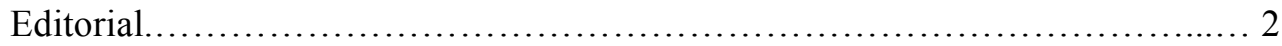

Nicole Naeve-Stoß, Julia Kastrup \& Gabriele Herold

Praxisphasen im Rahmen des Studiums Lehramt an beruflichen Schulen -

Gestaltung und Erfahrungen an der Universität Hamburg................................. 3

Sandra Mester

Das schulische Praxissemester in NRW: Konzept und Innovation am Institut

für Berufliche Lehrerbildung der Fachhochschule Münster....................... 17

Andrea Mohorič

Transfer von Modellversuchsergebnissen - ein Beispiel aus dem

Förderschwerpunkt Berufsbildung für nachhaltige Entwicklung

Karolin Wirth

Inklusion: Ansätze zur praktischen Umsetzung in der

beruflichen Lehrerbildung................................................. 40

Susanne Miesera \& Stefanie Sander

Sprache im Beruf in Bildungsgängen der beruflichen Fachrichtung

Ernährung und Hauswirtschaft.................................................... 54

Anna Hoff

Fachräume im berufsbildenden Unterricht - die Lehrküche als Beispiel

Helge Schulz

Bildungsgangarbeit in der Fachdidaktik

Alexandra Brutzer \& Christine Küster

Lernbereich Alltagskultur im Berufsfeld Ernährung und Hauswirtschaft -

Skizze für einen Orientierungsrahmen.

Julia Kastrup \& Irmhild Kettschau

Berufliche Lehrkräftebildung gestärkt -

Bundesarbeitsgemeinschaft gegründet. 108

Marie Nölle

Rezension: Berufsbildung für nachhaltige Entwicklung. 


\section{Transfer von Modellversuchsergebnissen - ein Beispiel aus dem Förderschwerpunkt Berufsbildung für nachhaltige Entwicklung}

Im Artikel wird am Beispiel der Berufsbildung für nachhaltige Entwicklung dargestellt, wie Modellversuchsergebnisse dauerhaft in die Bildungspraxis überführt werden können. Hierbei zeigt sich, dass es neben einer für alle relevanten Partner überzeugenden und tragfähigen Innovation vor allem auch auf die Passung zu vorhandenen Strukturen und Ordnungen ankommt.

Schlüsselworte: Transfer von Modellversuchsergebnissen, Weiterbildungskonzept, Berufsbildung für nachhaltige Entwicklung

\section{Einleitung}

Die Entwicklung eines Fort- oder Weiterbildungskonzepts ist ein oft gewähltes Ziel und Ergebnis von Modellversuchen und Innovationsentwicklungsprojekten. Ziel der Projekte und Anforderung der Fördergeber ist es, eine übertragbare Lösung zu entwickeln, die den Transfer in andere Regionen und/oder den Anschluss an das Berufsbildungssystem z. B. im Sinne der Eröffnung neuer Bildungs- und Karrierewege gewährleistet.

Am Beispiel des Fortbildungscurriculums Fachwirt/-in Erneuerbare Energien/Energieeffizienz (FEE) wird gezeigt, welche Elemente wichtig sind, damit ein am Markt nachgefragtes Konzept entsteht. Es wird thematisiert, welches Potenzial dieses Konzept für ein bundesweit anerkanntes Fortbildungsangebot besitzt, sowie welche weiteren Schritte dazu notwendig sind. Beleuchtet werden die Aufgaben und Rollen der Verbundpartner, die das Konzept entwickelt haben - der Kammern und des Zentralverbands des Deutschen Handwerks (ZDH) als zuständige Stellen zur Anerkennung und Standardisierung von Weiterbildungsangeboten sowie des Bundesinstituts für Berufsbildung (BIBB) in seiner Rolle als Vermittler und Transferakteur im Förderschwerpunkt.

\section{Inhalte und Potenziale des Fortbildungscurriculums}

Im Modellversuch „Fachwirt/in Erneuerbare Energie und Energieeffizienz“ entwickelten die Universität Oldenburg, die Handwerkskammer Oldenburg und das Bundestechnologiezentrum für Elektrotechnik in Oldenburg (bfe) die kammerzertifizierte 


\section{Transfer von Modellversuchsergebnissen}

Fortbildung „Fachwirt/in Erneuerbare Energie und Energieeffizienz“ (vgl. Rebmann, Schlömer, Feldkamp, Jancke \& Lüllau, 2014). Das Kompetenzprofil zur Gestaltung der Energiewende beinhaltet neben technischen, energiesystem- und energieträgerbezogenen Kompetenzen auch wirtschaftsbezogene Kompetenzen und Schlüsselqualifikationen. Es handelt sich um eine Fortbildung, die zwischen der Gesellen- und der Meisterstufe ansetzt und am betrieblichen Knotenpunkt zwischen Gewerken der Elektrotechnik und Anlagenmechanik angesiedelt ist. Kundenorientierung und Kundenberatung sowie Schnittstellenkompetenzen in der Kooperation der Gewerke sind dabei entscheidend. Wahlmodule bieten die Möglichkeit für regionale Differenzierung des Bildungsangebots.

Die konkreten praxisrelevanten Produkte sind:

- HWK-Prüfungsordnung und damit Verankerung als kammerzertifiziertes Weiterbildungsangebot mit anerkanntem Abschluss,

- handlungsorientiertes Curriculum (modularisiert, flexibel) der Fortbildung mit 28 Modulen (400 h), die in drei Felder gegliedert sind: Energieeffizienz, Erneuerbare Energien und kaufmännisches Management,

- Durchführungskonzept und Fortbildungsinfrastruktur, Selbstlernmaterialien, Prüfungsprojekt aus der Arbeitspraxis.

Der Fortbildungslehrgang erfreut sich großer Nachfrage und scheint genau den Bedarf zu treffen. Die gute Nachfrage von Teilnehmenden heterogener Berufsbiografien nach der Fortbildung bietet Anknüpfungspunkte zur Entwicklung einer bundeseinheitlichen Fortbildungsregelung.

\section{Anforderungen an den Transfer}

Die Verankerung von Modellversuchsergebnissen im Berufsbildungssystem ist von der Anschlussfähigkeit verschiedener Aspekte abhängig: Für Teilnehmende ist die Anerkennung einer besuchten Fortbildung entscheidend, damit der Abschluss (Zertifikat) auf dem Arbeitsmarkt und gegenüber Personalverantwortlichen wertgeschätzt und karriereförderlich ist (vgl. BIBB, 2013a, S. 24-29). Zudem ist wichtig, dass das Fortbildungsangebot, wenn es sich um eine Aufstiegsqualifizierung handelt, in die Systematik der vorgesehenen Qualifizierungsebenen passt, damit die Durchlässigkeit und Anrechnung auf folgende Qualifikationsebenen ermöglicht wird. Die Akkreditierung eines Fortbildungskonzepts gehört zur Mindest-Anforderung und trägt zur Marktpositionierung bei. Diese geprüften Konzepte können somit Eingang in berufliche Aus- und Weiterbildungsordnungen finden. Um eine Anerkennung bei einer Zertifizierungs- bzw. Akkreditierungsstelle zu erhalten, unterliegt ein Bildungskonzept entsprechenden Qualitätskriterien und muss systematisch dargestellt sein sowie definierte Prüfungsinhalte aufweisen (vgl. Mohorič, 2013). Nach dem Berufsbildungsgesetz sind die Kammern als zuständige Stellen mit der Regelung von Fortbildungsprüfungen im regionalen Raum betraut. Bei bundeseinheitlichen Fortbildungsordnungen ist der Bund, d. h. das jeweils zuständige der Ordnungsgeber. 
In der Systematik der Transferformen nach Kastrup, Kuhlmeier \& Reichwein (2014) ist dem Projekt der „,temporale Transfer“, also die Verstetigung in der eigenen Einrichtung und in den Strukturen vor Ort - hier durch eine Kammerprüfungsordnung -, gelungen (vgl. S. 175). Ein „regionaler Transfer“ gewährleistet die Anwendung des Konzepts in Weiterbildungseinrichtungen in anderen Regionen. Ein „vertikaler Transfer" könnte gelingen, wenn eine regelhafte Implementierung des Konzepts in übergeordneten Strukturen des Berufsbildungssystems erreicht wird.

\section{Elemente auf dem Weg zur bundeseinheitlichen Anerkennung eines Fortbildungskonzepts}

Ein nachgefragtes Weiterbildungskonzept sollte folgende Voraussetzungen erfüllen, um eine Passfähigkeit in der beruflichen Weiterbildung zu gewährleisten:

- Fortbildungsprüfungsregelung mit lokaler Geltung

Beim Projekt Fachwirt/in war die örtliche Handwerkskammer Oldenburg als Projektpartner eingebunden und wirkte bei der Entwicklung des Konzepts mit, was dazu führte, dass eine Prüfungsordnung nach der Handwerksordnung entwickelt wurde. Zum einen spricht dies für die Einbindung von Unternehmensbedarfen in die Konzeptentwicklung, zum andern entspricht das Fortbildungsangebot bestimmten Qualitätsanforderungen, die mit einer solchen Rechtsverordnung verbunden sind: Die Fortbildungsprüfung zum $/ \mathrm{r}$ Fachwirt/in Erneuerbare Energien/Energieeffizienz (HWK) beinhaltet die Bezeichnung des Abschlusses, das Ziel der Prüfung, die Zulassungsvoraussetzungen, Gliederung, Inhalt und Dauer der Prüfung, Anrechnung der Prüfungsleistungen und das Prüfungsverfahren.

- Bundeseinheitliche Fortbildungsordnung

Voraussetzung für eine bundeseinheitliche Regelung ist ein nachweisbarer qualitativer und quantitativer überregionaler Bedarf auf dem Arbeitsmarkt. Die Regelung ist zeitlich nicht begrenzt. Die Fortbildung sollte zur beruflichen Mobilität und vielseitigen Einsetzbarkeit der Berufstätigen beitragen und klar zur Berufsausbildung abgrenzbar. Die Voraussetzungen für eine bundeseinheitliche Fortbildungsordnung sind: eine Aufstiegsfortbildung mit bestehender fünfjähriger Kammerregelung, in fünf Bundesländern umgesetzt und 500 Teilnehmende in den letzten drei Jahren. Allerdings können die Spitzenorganisationen der Sozialpartner im Einzelfall diese Voraussetzungen außer Kraft setzen, wenn ein spezieller begründeter Bedarf für eine Aufstiegsfortbildung vorliegt. Sie erlassen dann im Rahmen einer DHKTEmpfehlung eine bundeseinheitliche Fortbildungsordnung.

- Anschlussfähigkeit der Aufstiegsqualifizierung an die DQR-Niveaus Wichtiges Anliegen des Deutschen Qualifizierungsrahmens (DQR) ist es, die Transparenz und Durchlässigkeit zwischen den Bildungsbereichen - insbesondere der nicht-akademischen Bildung gegenüber akademischer Bildung - 


\section{Transfer von Modellversuchsergebnissen}

zu fördern. Die folgenden Angaben geben die Beziehung zwischen Ebenen bzw. Umfängen einer Aufstiegsqualifizierung und den DQR-Niveaus an:

Ebene 1/DQR-Niveau 5: z. B. Fachberater/in mit mindestens $200 \mathrm{~h}$ - Erweiterung/Vertiefung der erworbenen beruflichen Kompetenzen.

Ebene 2/DQR-Niveau 6: z. B. Fachwirt/in mit $500-600$ h - mittlere Führungsebene und erweiterter Verantwortungsbereich.

Ebene 3/DQR-Niveau 7: z. B. Betriebswirt/in mit über $600 \mathrm{~h}$ - leitende Führungsposition eines Unternehmens/-bereichs als Alternative zu Hochschulabschlüssen (vgl. BIBB, 2013b, S. 24-28; Born 2014).

\section{Anschluss an das berufliche Weiterbildungssystem}

Das vorliegende Fortbildungskonzept bietet zwei Voraussetzungen für eine bundeseinheitliche Reglung: die Nachfrage und die überregionale Verbreitung. Für die neuentwickelte Generalisten-Fortbildung „Fachwirt/in Erneuerbare Energie und Energieeffizienz" mit Kammerprüfung startet mittlerweile der dritte Lehrgang (finanziell selbsttragend), der sehr gut von Teilnehmenden diverser Berufe angenommen wird. Partnerorganisationen stehen für eine überregionale Ausdehnung des Konzeptes zur Verfügung. Zudem haben vier weitere Handwerkskammern hohes Interesse daran, den Fortbildungslehrgang in Ihr Angebot aufzunehmen Es ist allerdings davon abhängig, wie sich das Fortbildungskonzept in die Systematik der Aufstiegsqualifizierung nach DQR einfügt, was Stundenvolumina und Niveaustufen anbetrifft. In dieser Systematik beträgt eine Fachwirt/in-Qualifizierung $600 \mathrm{~h}$ im Gegensatz zum entwickelten Konzept, das 400 h umfasst; damit ist die Anschlussfähigkeit nicht vollständig gegeben. Hier müsste eine Anpassung des Konzepts erfolgen.

\section{Passfähigkeit von Weiterbildungskonzepten}

Die Rollen und Aufgaben der Berufsbildungsakteure sind hinsichtlich des temporalen, regionalen und vertikalen Transfers unterschiedlich.

\section{Projekt-/Verbundpartner}

Ein interdisziplinäres Projekt-Konsortium und/oder ein begleitender Projektbeirat gewährleisten, dass alle wichtigen Berufsbildungsakteure bei der Entwicklung eingebunden sind und begleitend beraten. Hier ist auch wichtig, die unterschiedlichen Strategien und Interessen, die auf dem Weiterbildungsmarkt und im Bereich der Ordnungsmittel eine Rolle spielen, zur Kenntnis zu nehmen, um daraus Entscheidungen und Schlussfolgerungen für die eigene Weiterbildungskonzeption zu ziehen. Außerdem zeigt sich der Nutzen einer wissenschaftlich fundierten Bedarfsanalyse dahingehend, dass die Nachfrage zielgenauer getroffen werden kann. Beim Fachwirt/in Erneuerbare Energie/Energieeffizienz hat die Universität Oldenburg eine qualitative und quantitative Erforschung der Geschäftsmodelle in der dezentralen 


\section{Transfer von Modellversuchsergebnissen}

Energiewirtschaft und des Berufshandelns in den Betrieben der Energiewirtschaft vollzogen (vgl. Rebmann et al., 2014). Auf dieser Grundlage wurde in enger gemeinsamer Entwicklungsarbeit von Forschung und Praxis ein erfolgreiches Fortbildungskonzept formuliert. Damit ist ein forschungsbasiertes bedarfsorientiertes Fortbildungskonzept für die Energiewende gelungen.

\section{Zentralverband des deutschen Handwerks (ZDH)}

Als Spitzenorganisation des deutschen Handwerks ist der ZDH an Qualitätsstandards und einer systematischen beruflichen Weiterbildung interessiert. Er vertritt mit dem Berufslaufbahnkonzept eine bildungspolitische Strategie in der Fortbildung, die Aufstiegsqualifizierungswege (auf den DQR-Stufen 5, 6,7) für Fach- und Führungskräfte vorsieht und (weiter)entwickeln möchte. Der ZDH zeigte sich offen und interessiert an dem Konzept Fachwirt/-in Erneuerbare Energien/Energieeffizienz (FEE) und sieht begründetes Potential für eine DHKT-Empfehlung, vorausgesetzt es folgt dieser Systematik..

\section{Bundesinstitut für Berufsbildung (BIBB)}

Das BIBB nimmt bei der Weiterentwicklung von Ordnungsmitteln der Berufsbildung die wichtige Rolle als Moderator und Koordinator wahr. Im Fall der Fortbildungsordnungen ist das BIBB verantwortlich für das Ordnungsverfahren und den Entwurf der bundesweiten Fortbildungsordnung. Beim Fachwirt/in Erneuerbare Energien/Energieeffizienz ging es zum einen darum, die Gremien des ZDH auf das Fortbildungskonzept aufmerksam zu machen. Zum andern war auszuloten, inwieweit das Potenzial des Konzepts ausreicht, um zu einer bundeseinheitlichen Fortbildungsregelung zu werden, bzw. welche Voraussetzungen und Weiterentwicklungen des Konzepts dafür erforderlich sind. In Förderprogrammen kann der Transfer von Weiterbildungskonzepten in das Berufsbildungssystem gestärkt werden, wenn die Passfähigkeit zu den Ordnungsmitteln, die Qualität von akkreditierten/zertifizierten Konzepten und die Anschlussfähigkeit an berufliche Aufstiegswege und Qualifizierungssystematiken beachtet wird. Bei dem Beispiel des/der Fachwirt/in Erneuerbare Energien/Energieeffizienz bieten die aktuellen Ergebnisse strukturbildende Ansatzpunkte zum Transfer in Ordnungsmittel und damit zu einer festen Verankerung im Bildungssystem an.

\section{Fazit}

Bei der Entwicklung von Fortbildungskonzepten ist die Reichweite, Verbreitung und Übertragung der Projektergebnisse zu beachten, wenn eine Verstetigung von Konzepten durch den Transfer in die Ordnungsmittel gewährleistet sein soll. Strukturbildende Ansatzpunkte in der beruflichen Weiterbildung sind für einen zeitlich befristeten Transfer dann gegeben, wenn der Projektverbund eine Akkreditierung der zuständigen Stelle anstrebt und in die Konzeptentwicklung einbezieht. Ideal ist - wie in unserem Beispiel geschehen -, wenn die örtliche Handwerkskammer im Projektver- 


\section{Transfer von Modellversuchsergebnissen}

bund beteiligt ist. Der Erfolg des Fortbildungsangebots ist marktabhängig, insofern ist eine solide Bedarfsanalyse als Grundlage der Entwicklungsarbeit unerlässlich. Forschungsbasierte Modellversuche haben sich für diesen Praxis-WissenschaftPolitik-Dialog bei der Entwicklung von Konzepten bewährt. Ein regionaler Transfer - wie unser Beispiel zeigt - gelingt durch eine Verbundstruktur bzw. Netzwerke, die das Konzept in anderen Regionen implementieren. Soll das Konzept bundesweite Anerkennung und Verbreitung finden und so ein vertikaler Transfer ins Bildungssystem zustande kommen, ist eine möglichst eindeutige Einordnung in die Systematik der Aufstiegsqualifizierung notwendig. Nur so sind Anschlussfähigkeit an verschiedene Qualifikationsniveaus und Durchlässigkeit gegeben sowie ein Transfer gesichert.

\section{Literatur}

Bundesinstitut für Berufsbildung BIBB. (Hrsg.). (2013a). Checkliste - Qualität beruflicher Weiterbildung.

[www.bibb.de/veroeffentlichungen/de/publication/show/id/2061].

Bundesinstitut für Berufsbildung BIBB (Hrsg.). (2013b). Fortbildungsordnungen und wie sie entstehen.

[www.bibb.de/veroeffentlichungen/de/publication/show/id/7062].

Kastrup, J., Kuhlmeier, W., Reichwein, W. (2014). Der Transfer der Ergebnisse des Förderschwerpunkts „Berufsbildung für eine nachhaltige Entwicklung“: Erfahrungen, Modell und Empfehlungen. In W. Kuhlmeier, A. Mohorič, Th. Vollmer (Hrsg.), Berufsbildung für nachhaltige Entwicklung - Modellversuche 20102013: Erkenntnisse, Schlussfolgerungen und Ausblicke (S. 171-181). Bielefeld: Bertelsmann.

Mohorič, A. (2013). Thematisches Monitoring: Professionalisierung des Bildungspersonals. Thesenpapier-Collage: Wandel der Bildungsberufe und Professionalisierungsstrategien - Thematisches Monitoring europäischer Projekte.

[www.na-bibb.de/fileadmin/user_upload/Dokumente/ EU/Bildungspersonal/Thesenpapier_Professionalisierung.pdf].

Mohorič, A. (2014). Berufsbildung für nachhaltige Entwicklung - Das Bundesinstitut für Berufsbildung als Akteur und Moderator bei der Gestaltung des Transfers von Modellversuchsergebnissen. In W. Kuhlmeier, A. Mohorič, \& Th. Vollmer (Hrsg.), Berufsbildung für nachhaltige Entwicklung - Modellversuche 20102013: Erkenntnisse, Schlussfolgerungen und Ausblicke (S. 183-196). Bielefeld: Bertelsmann.

Mohorič, A. (2003). Transferprobleme von Modellprojekten/-versuchen in Organisationen - ein Lösungsversuch. Neue Perspektiven, Zeitschrift für berufliche Bildung und Weiterbildung, 1, 5-17.

Rebmann, K., Schlömer, T., Feldkamp, D., Jancke, H. \& Lüllau, Ch. (2014). Das Oldenburger Modell der Berufsbildung für nachhaltige Entwicklung und seine Ausgestaltung im Modellversuch der Fortbildung zur Fachwirtin/zum Fachwirt 


\section{Transfer von Modellversuchsergebnissen}

Erneuerbare Energien Energieeffizienz (HWK). In W. Kuhlmeier, A. Mohorič \& Th. Vollmer (Hrsg.), Berufsbildung für nachhaltige Entwicklung - Modellversuche 2010-2013: Erkenntnisse, Schlussfolgerungen und Ausblicke (S. 69-93). Bielefeld: Bertelsmann.

\section{Verfasserin}

Andrea Mohorič

Programmleiterin Modellversuchsschwerpunkt Berufsbildung

für nachhaltige Entwicklung am Bundesinstitut für Berufsbildung (BIBB)

E-Mail: mohoric@bibb.de 